\title{
Arachidonic acid and DHA status in pregnant women is not associated with cognitive performance of their children at 4 or 6-7 years
}

\author{
Sarah R. Crozier ${ }^{1}$, Charlene M. Sibbons ${ }^{2}$, Helena L. Fisk ${ }^{2}$, Keith M. Godfrey ${ }^{1,2,3}$, Philip C. Calder ${ }^{2,3}$, \\ Catharine R. Gale ${ }^{1,4}$, Sian M. Robinson ${ }^{1,3}$, Hazel M. Inskip ${ }^{1,3}$, Janis Baird ${ }^{1,3}$, Nicholas C. Harvey ${ }^{1,3}$, \\ Cyrus Cooper ${ }^{1,3}$, Graham C. Burdge ${ }^{2 *}$ and Southampton Women's Survey (SWS) Study Group ${ }^{1}$ \\ ${ }^{1}$ MRC Lifecourse Epidemiology Unit, Southampton General Hospital, University of Southampton, Southampton SO16 6YD, UK \\ ${ }^{2}$ Academic Unit of Human Development and Health, Faculty of Medicine, University of Southampton, Southampton SO16 6YD, UK \\ ${ }^{3}$ NIHR Southampton Biomedical Research Centre, University Hospital Southampton NHS Foundation Trust and University \\ of Southampton, Southampton SO16 6YD, UK \\ ${ }^{4}$ Department of Psychology, Centre for Cognitive Ageing \& Cognitive Epidemiology, University of Edinburgh, Edinburgh EH8 \\ 9JZ, UK \\ (Submitted 17 July 2017 - Final revision received 1 March 2018 - Accepted 5 March 2018 - First published online 8 May 2018)
}

\section{Abstract}

Arachidonic acid (ARA) and DHA, supplied primarily from the mother, are required for early development of the central nervous system. Thus, variations in maternal ARA or DHA status may modify neurocognitive development. We investigated the relationship between maternal ARA and DHA status in early ( 11.7 weeks) or late ( 34.5 weeks) pregnancy on neurocognitive function at the age of 4 years or 6-7 years in 724 mother-child pairs from the Southampton Women's Survey cohort. Plasma phosphatidylcholine fatty acid composition was measured in early and late pregnancy. ARA concentration in early pregnancy predicted $13 \%$ of the variation in ARA concentration in late pregnancy $(\beta=0.36$, $P<0 \cdot 001)$. DHA concentration in early pregnancy predicted $21 \%$ of the variation in DHA concentration in late pregnancy $(\beta=0 \cdot 46, P<0 \cdot 001)$. Children's cognitive function at the age of 4 years was assessed by the Wechsler Preschool and Primary Scale of Intelligence and at the age of 6-7 years by the Wechsler Abbreviated Scale of Intelligence. Executive function at the age of 6-7 years was assessed using elements of the Cambridge Neuropsychological Test Automated Battery. Neither DHA nor ARA concentrations in early or late pregnancy were associated significantly with neurocognitive function in children at the age of 4 years or the age of $6-7$ years. These findings suggest that ARA and DHA status during pregnancy in the range found in this cohort are unlikely to have major influences on neurocognitive function in healthy children.

Keywords: Arachidonic acid: DHA: Pregnancy: Intelligence quotient: Neurocognition

The PUFA arachidonic acid (ARA) and DHA are major components of neural cell membrane phospholipids ${ }^{(1,2)}$. In humans, there is substantial accumulation of ARA and DHA into the fetal brain during the third trimester of pregnancy ${ }^{(1,2)}$. The human fetus is dependent largely on the transfer of preformed ARA and DHA from the mother across the placenta. Human term infants fed milk formula without preformed DHA exhibit low DHA concentrations in the brain $^{(3)}$ and plasma phospholipids ${ }^{(4)}$. Studies in non-human primates have shown that maternal diets deficient in $n-3$ PUFA are associated with impaired cognition and abnormal behaviour in their offspring ${ }^{(5,6)}$. It is therefore considered important to ensure adequate provision of DHA and ARA during brain development ${ }^{(7)}$.

There have been relatively few studies of the effect of maternal or neonatal ARA and DHA status on neurocognitive function in children. ARA and DHA status at birth has been shown not to be associated with cognitive development at the age of 4 years $^{(8)}$, or with problem behaviour ${ }^{(9)}$ and cognitive development $^{(10)}$ at the age of 7 years, although there was a positive association with motor function ${ }^{(10)}$. In contrast, maternal fish intake, a proxy measure of DHA intake, was positively associated with developmental milestones at 6 and 18 months ${ }^{(11)}$ and with cognition at the age of 3 years ${ }^{(12)}$. Maternal sea food intake has also been positively associated with verbal intelligence quotient (IQ) in children ${ }^{(13)}$, although others have concluded that maternal fish intake during pregnancy had little long-term effect on the neurodevelopment of the child ${ }^{(14)}$. However, these studies did not report maternal ARA or DHA status.

The primary purpose of the present study was to determine the relationship between maternal ARA and DHA concentrations in early and late pregnancy, and neurocognitive outcomes

Abbreviations: ARA, arachidonic acid; IED, intra/extra-dimensional shift; IQ, intelligence quotient; PC, phosphatidylcholine; SWS, Southampton Women's Survey. 
in their children at the age of 4 years or at the age of 6-7 years. PUFA concentrations were measured at two time points in gestation because DHA concentration increases physiologically from mid pregnancy ${ }^{(15-17)}$ owing to adaptions to maternal hepatic phospholipid ${ }^{(18)}$ and PUFA metabolism ${ }^{(19)}$. We also tested the relationship between the change in ARA and DHA status during pregnancy, as a surrogate measure of the mother's capacity to adapt her PUFA metabolism, and neurocognitive function in children.

\section{Methods \\ Ethical statement}

The Southampton Women's Survey (SWS) was approved by the Southampton and South West Hampshire Local Research Ethics Committee (307/97, 153/99w, 005/03/t and 06/Q1702/104), and all participants gave written informed consent.

\section{Study sample}

The SWS is a prospective cohort study of the impact of the early life environment on patterns of health throughout the life course in which the diet, body composition, physical activity and social circumstances of non-pregnant women aged 20-34 years living in the city of Southampton, UK, were characterised $^{(20)}$. Women were recruited through primary healthcare practices across the city between April 1998 and December 2002. Women who subsequently became pregnant with singleton fetuses were followed up throughout pregnancy; detailed interviews were conducted at 11 and 34 weeks of gestation, when blood samples were collected for fatty acid analysis after an overnight fast. The growth and development of the SWS children were assessed during infancy and childhood.

A total of 3158 women became pregnant and delivered a liveborn singleton infant within the study period (online Supplementary Fig. S1). Eight infants died in the neonatal period. Subsets of children were followed up at the age of 4 years and at the age of 6-7 years. A total of 1207 offspring had data collected about cognitive development at the age of 4 years or at the age of 6-7 years. In all, 724 mothers did not have exposure data on plasma phosphatidylcholine (PC) fatty acid composition, leaving an analysis sample of 724 mother-child pairs. Of these, 584 gave blood samples in early pregnancy and 331 gave blood samples in late pregnancy. A total of 191 women provided blood samples in early pregnancy (median 11.7 (interquartile range (IQR) 11.4, 12.2) weeks), before the start of the physiological increase in plasma PC DHA concentration ${ }^{(15)}$ and in late (median 34.5 (IQR 34.2, 34.8) weeks) pregnancy, corresponding to maximum plasma PC DHA concentration ${ }^{(15)}$. Details of mothers' educational attainment (defined in six groups according to highest academic qualification) were obtained at the pre-pregnancy interview. Height was measured with a portable stadiometer (Harpenden; CMS Weighing Equipment Ltd) to the nearest $0.1 \mathrm{~cm}$ with the head in the Frankfort plane. Weight was measured using calibrated electronic scales (Seca) to the nearest $0 \cdot 1 \mathrm{~kg}$ (after removal of shoes and heavy clothing or jewellery). These measurements were used to calculate BMI. Among women who became pregnant, smoking status was ascertained. Maternal IQ was assessed when her children were aged 4 years and 6-7 years using the Wechsler Abbreviated Scale of Intelligence (WASI).

\section{Maternal sample collection and plasma fatty acid composition}

Venous blood samples were collected into tubes containing lithium heparin in early and late pregnancy. Plasma was separated from cells by centrifugation and stored at $-80^{\circ} \mathrm{C}$. Plasma PC fatty acid composition was measured essentially as described ${ }^{(21)}$. In brief, frozen plasma $(0.8 \mathrm{ml})$ was thawed, dipentadecanoyl PC $(100 \mu \mathrm{g})$ internal standard was added and total lipids were then extracted with chloroform and methanol. Lipid extracts were dried under $\mathrm{N}_{2}$, dissolved in chloroform $(1.0 \mathrm{ml})$ and applied to a BondElut aminopropylsilica cartridge $(100 \mathrm{mg}$ ) (Agilent Technologies). Unbound lipids were removed by washing with chloroform, and PC was then eluted with chloroform-methanol (60:40, $\mathrm{v} / \mathrm{v}$ ). Purified PC was dissolved in toluene, and fatty acid methyl esters (FAME) were synthesised by heating at $50^{\circ} \mathrm{C}$ in the presence of methanol containing $2 \%(\mathrm{v} / \mathrm{v})$ sulphuric acid. FAME were recovered with hexane and resolved on a BPX-70 fused silica capillary column $(32 \mathrm{~m} \times 0.25 \mathrm{~mm} \times 25 \mu \mathrm{m}$; SGE Analytical Science) using an Agilent 6890 gas chromatograph equipped with flame ionisation detection (Agilent Technologies Ltd). The concentrations of ARA and DHA were calculated from the ratio of their peak areas to the peak area of the internal standard, multiplied by the amount of standard and corrected for the volume of plasma extracted.

\section{Assessment of cognitive function in children}

IQ was assessed at age 4 years using the Wechsler Preschool and Primary Scale of Intelligence (WPPSI) ${ }^{(22)}$ and at age 6-7 years using the WASI ${ }^{(23)}$. Executive functioning was tested at the age of 6-7 years using the Cambridge Neuropsychological Test Automated Battery $\left(\mathrm{CANTAB}^{\circledR}\right)$, with four specific tests and outcomes chosen based on the published literature: these were (1) delayed matching to sample (DMS) (i) total correct, to test visual working memory; (2) intra/extradimensional shift (IED), (ii) total errors, (iii) adjusted errors, and (iv) stages completed, to test rule learning and cognitive flexibility through efficiency of completing the test, (3) Spatial Span (SSP) length, to test working memory), and (4) Information Sampling Task (IST), (vi) pre-extradimensional shift errors, (vii) extradimensional shift errors and (viii) adjusted IED total errors, to test impulsivity and decision making ${ }^{(24)}$.

\section{Statistical analysis}

Children's IQ was the primary study outcome for which we calculated the statistical power of the analysis. In all, 260 participants had IQ measured at 4 years; of these, 146 participants had measures of early pregnancy fatty acid status and 253 had measures of late pregnancy fatty acid status. As these were all the participants in the SWS cohort with these measurements, further data collection is not feasible. Consequently, we have 
determined minimally detectable effect sizes. Our calculations show that these numbers have $80 \%$ power to detect regression coefficients of 2.9 and 2.2 at a $5 \%$ significance level, in early and late pregnancy, respectively. Thus, we had sufficient numbers to detect a change in IQ of 2.9 (or 2.2) points for each standard deviation change in maternal fatty acid status. An increase in IQ of 2.9 or 2.2 points equates to a change in the distribution of IQ in a favourable direction of approximately $0 \cdot 2$ of a standard deviation (based on the standard deviation at age 4). This difference in IQ would have only a modest impact at an individual level. However, according to Rose's theory of prevention ${ }^{(25)}$, a shift in the population mean IQ of that magnitude would potentially have a marked effect on cognitive ability in that population as it would prevent many individuals from having cognitive problems.

Summary statistics are presented as means and standard deviations or medians and IQR for continuous variables and percentages for categorical variables. $t$ Tests (for normally distributed continuous variables), Mann-Whitney $U$ tests (for non-normally distributed continuous variables) and $\chi^{2}$ tests (for categorical variables) were used to compare the distributions of characteristics between omnivores and vegetarians. Maternal ARA and DHA levels, and changes in DHA and ARA concentrations in both early and late pregnancy, were log-transformed to normality before analysis. To assist with their interpretation, these logged variables were standardised so that the variables have an SD of 1. Maternal BMI was also log-transformed before analysis. Additional analyses used maternal ARA and DHA without transformation.

IED pre-extra-dimensional shift (EDS) errors, IED EDS errors and IED total errors (adjusted) were all transformed using Fisher-Yates transformations ${ }^{(26)}$, so the resulting variable has SD units. It was not possible to transform IED total errors (stage 1), IED total errors (stage 8) and IST mean probability correct, and thus these were divided into five groups. Similarly, the IED stages completed was grouped into four groups (five groups were inappropriate here owing to the distribution of responses). It was not necessary to transform DMS total correct or SSP span length, and thus these are in original units.

Linear regression models were fitted to assess the association between dietary exposures and cognitive development outcomes. Models were fitted unadjusted and adjusted for confounders. We used the directed acyclic graph (DAG) approach $^{(27)}$ to select suitable confounders (online Supplementary Fig. S2). This approach provides a robust and objective means of identifying confounders in observational studies. DAG are specified before data analysis based on prior knowledge. A graphical representation of causal effects between variables is generated in order to identify a set of variables that should be adjusted for in a multivariate analysis to minimise confounding bias $^{(27)}$. The confounders identified by the DAG for the association between maternal fatty acid status and childhood cognitive development were maternal BMI, maternal IQ, maternal education and maternal smoking. In addition, all models were adjusted for maternal BMI, maternal IQ and maternal smoking and for child's sex, and in the case of the CANTAB outcomes, age (the WASI and WPSSI outcomes are already adjusted for age), in order to improve the precision of the models.

\section{Results}

Phosphatidylcholine arachidonic acid and DHA concentrations in pregnant women

Maternal ARA concentration was 34\% lower in late pregnancy $(P=0.004)$ than in early pregnancy (Table 1$)$. DHA concentration was $32 \%$ lower in late pregnancy than in early pregnancy, although this did not reach statistical significance (Table 1). Maternal ARA and DHA concentrations in early pregnancy were significantly correlated with their concentrations in late pregnancy (both $P<0.001$ ) such that ARA concentration in early pregnancy predicted $13 \%$ of the variation in ARA concentration in late pregnancy $(\beta=0 \cdot 36)$, and DHA concentration in early pregnancy predicted $21 \%$ of the variation in DHA concentration in late pregnancy $(\beta=0 \cdot 46)$.

\section{The relationship between arachidonic acid and DHA concentration in maternal plasma phosphatidylcholine and cognitive function in their children}

Unadjusted and adjusted data are summarised in Tables 2-3. There were no significant associations between maternal ARA concentrations in early or late pregnancy and the WPPSI IQ composite IQ score adjusted or unadjusted at the age of 4 years (Table 2).

There were no significant associations between maternal ARA concentration in early or late pregnancy and any of the measures of cognitive function in the children at 6-7 years of age after adjustment for confounders (Table 2).

There were no significant associations between maternal plasma PC DHA concentration in early or late pregnancy, and the change in DHA concentration between early and late pregnancy, and cognitive function in the children at either 4 years or 6-7 years of age after adjustment for confounders (Table 3).

In additional analyses, untransformed maternal ARA and DHA were considered as predictors of offspring IQ at both 4 and 6 years of age (online Supplementary Table S1); none of the associations were statistically significant. These findings are exemplified as follows: a $10-\mu \mathrm{g} / \mathrm{ml}$ increase in early pregnancy ARA was associated with a -0.37 IQ point decrease $(95 \% \mathrm{CI}$ $-0.80,0.07)$ at the age of 4 years $(P=0 \cdot 10)$, whereas a $10-\mu \mathrm{g} / \mathrm{ml}$ increase in early pregnancy DHA was associated with a -0.03 (95\% CI $-0.80,0.74)$ IQ point decrease at the age of 4 years $(P=0.94)$

\section{Discussion}

The findings of this study quantify for the first time a modest association between maternal ARA and DHA concentrations in early and late pregnancy. However, there were no statically significant associations between maternal ARA or DHA concentrations during pregnancy, and their children's IQ or executive function.

The human fetus accumulates LC PUFA throughout gestation, although this occurs most rapidly during the last 5 weeks ${ }^{(1)}$ and is dependent primarily on supply of preformed ARA and DHA from the mother. Deprivation of $n$-3 PUFA during pregnancy in 
Table 1. Characteristics of 724 mothers and children studied ${ }^{*}$ (Medians and interquartile ranges (IQR); mean values and standard deviations; numbers and percentages)

\begin{tabular}{|c|c|c|c|}
\hline & \multicolumn{3}{|c|}{ Mother-child pairs studied } \\
\hline & $n$ & Median & IQR \\
\hline \multicolumn{4}{|l|}{ Mother } \\
\hline Age at child's birth (years) & 724 & & \\
\hline Mean & & \multicolumn{2}{|c|}{$31 \cdot 1$} \\
\hline SD & & \multicolumn{2}{|c|}{3.6} \\
\hline Educational attainment; qualifications $\geq A$-level & 723 & \multicolumn{2}{|c|}{456} \\
\hline$n$ & & \multirow{2}{*}{\multicolumn{2}{|c|}{$\begin{array}{l}456 \\
63 \cdot 1\end{array}$}} \\
\hline$\%$ & & & \\
\hline$I Q$, child age 4 years & 260 & \multirow{2}{*}{\multicolumn{2}{|c|}{$107 \cdot 8$}} \\
\hline Mean & & & \\
\hline SD & & \multicolumn{2}{|c|}{$12 \cdot 6$} \\
\hline $\mathrm{IQ}$, child age 6 years & 458 & \multirow{2}{*}{\multicolumn{2}{|c|}{$104 \cdot 2$}} \\
\hline Mean & & & \\
\hline SD & & \multicolumn{2}{|c|}{$15 \cdot 8$} \\
\hline Smoked in pregnancy & 712 & & \\
\hline$n$ & & \multicolumn{2}{|c|}{110} \\
\hline$\%$ & & \multicolumn{2}{|c|}{$15 \cdot 5$} \\
\hline $\mathrm{BMI}\left(\mathrm{kg} / \mathrm{m}^{2}\right)$ & 722 & $24 \cdot 4$ & $21 \cdot 9,27 \cdot 3$ \\
\hline Multiparous & 724 & \\
\hline$n$ & & \multirow{2}{*}{\multicolumn{2}{|c|}{$\begin{array}{c}312 \\
43 \cdot 1\end{array}$}} \\
\hline$\%$ & & & \\
\hline Duration breast-feeding (weeks) & 688 & $13 \cdot 0$ & $1.4,30.4$ \\
\hline Early-pregnancy plasma ARA concentration $(\mu \mathrm{g} / \mathrm{ml})$ & 584 & 172 & 142,212 \\
\hline Late-pregnancy plasma ARA concentration ( $\mu \mathrm{g} / \mathrm{ml})$ & 331 & 113 & 86,147 \\
\hline Early-pregnancy plasma DHA concentration $(\mu \mathrm{g} / \mathrm{ml})$ & 584 & 86 & 67,107 \\
\hline Late-pregnancy plasma DHA concentration $(\mu \mathrm{g} / \mathrm{ml})$ & 331 & 58 & 44,77 \\
\hline \multicolumn{4}{|l|}{ Child } \\
\hline Female & 724 & & \\
\hline$n$ & & \multirow{2}{*}{\multicolumn{2}{|c|}{$\begin{array}{c}346 \\
47.8\end{array}$}} \\
\hline$\%$ & & & \\
\hline Gestation at birth (weeks) & 724 & $40 \cdot 0$ & $39 \cdot 0-41 \cdot 0$ \\
\hline BMI at 4 years $\left(\mathrm{kg} / \mathrm{m}^{2}\right)$ & 260 & $15 \cdot 9$ & $15 \cdot 1-16 \cdot 7$ \\
\hline BMl at $6-7$ years $\left(\mathrm{kg} / \mathrm{m}^{2}\right)$ & 411 & $15 \cdot 7$ & $14.9,16 \cdot 9$ \\
\hline Age at 4 years (years) & 260 & & \\
\hline Mean & & \multicolumn{2}{|c|}{4.4} \\
\hline SD & & \multicolumn{2}{|c|}{0.1} \\
\hline Age at $6-7$ years (years) & 419 & & \\
\hline Mean & & \multicolumn{2}{|c|}{$7 \cdot 0$} \\
\hline SD & & \multicolumn{2}{|c|}{0.2} \\
\hline
\end{tabular}

* Sample sizes vary owing to outcome-specific missing values.

non-human primates has been shown to induce impaired neurological development in their offspring ${ }^{(5)}$. Thus, it may be anticipated that variation in maternal ARA and DHA status, particularly during the third trimester, would be associated with differences in neurocognitive development. Previous studies have shown longitudinal changes in DHA and ARA concentrations during pregnancy ${ }^{(15,16)}$. However, they did not report the relationship between maternal DHA or ARA status in early and late gestation. Both studies showed an increase in DHA concentration between early and late gestation, with the exception of Hungarian and Ecuadorian cohorts $^{(16)}$. In contrast to cohorts studied previously in the $\mathrm{UK}^{(15,16)}$, we found that maternal plasma ARA and DHA concentrations decreased during pregnancy by 34 and $32 \%$, respectively, although this change in DHA was not significant. The reason for this decrease could not be deduced from the present data. However, these findings suggest a reduction in capacity to supply these PUFA to the developing fetus during a period in which the developing brain acquires substantial amounts of ARA and DHA ${ }^{(1)}$.
The present study reports for the first time that there were no significant associations between maternal ARA and DHA status in early or late pregnancy, and measures of executive function and IQ in children. These findings suggest that, within the range of this cohort, variation in concentrations of these fatty acids in maternal blood during pregnancy exerts at most a minor influence on neurocognitive development in children. This suggestion is supported by the findings of studies in which pregnant women took a DHA supplement during pregnancy, which showed no significant effect on psychomotor, mental development or behavioural scores at 18 months ${ }^{(28,29)}$, or on executive function at the age of 2 years ${ }^{(29)}$. However, others have reported improved attention at the age of 5 years ${ }^{(30)}$. Moreover, a systematic review of eight randomised controlled trials failed to detect a significant effect of maternal supplementation with DHA during breast-feeding on neurocognitive outcomes $^{(31)}$. However, because this study did not investigate the nutrition of the children in the period between birth and the ages at which they were studied, postnatal dietary intakes of preformed ARA and DHA may have ameliorated any deficit in 
Table 2. Maternal plasma phosphatidylcholine arachidonic acid concentration as a predictor of cognitive outcomes (Linear regression coefficients $(\beta)$ and $95 \%$ confidence intervals)

\begin{tabular}{|c|c|c|c|c|c|c|c|c|}
\hline & \multicolumn{4}{|c|}{ Unadjusted } & \multicolumn{4}{|c|}{ Adjusted } \\
\hline & $\beta$ & $95 \% \mathrm{Cl}$ & $P$ & $n$ & $\beta$ & $95 \% \mathrm{Cl}$ & $P$ & $n$ \\
\hline \multicolumn{9}{|l|}{4 years WPPSI IQ } \\
\hline Early pregnancy (SD) & $-2 \cdot 24$ & $-4.56,0.07$ & 0.06 & 146 & -2.00 & $-4.33,0.34$ & 0.09 & 146 \\
\hline Late pregnancy $(\mathrm{SD})$ & 0.40 & $-1 \cdot 22,2 \cdot 02$ & 0.63 & 253 & 0.39 & $-1 \cdot 16,1.94$ & 0.62 & 253 \\
\hline Late-early pregnancy change (SD) & $3 \cdot 10$ & $0.36,5.85$ & 0.03 & 139 & $2 \cdot 74$ & $-0.05,5.52$ & 0.05 & 139 \\
\hline \multicolumn{9}{|l|}{$6-7$ years WASI } \\
\hline Early pregnancy (SD) & -0.30 & $-1 \cdot 74,1 \cdot 14$ & 0.68 & 432 & 0.14 & $-1 \cdot 18,1.45$ & 0.84 & 414 \\
\hline Late pregnancy (SD) & -2.15 & $-6 \cdot 53,2.22$ & 0.33 & 77 & -1.27 & $-5.78,3.25$ & 0.58 & 76 \\
\hline Late-early pregnancy change (sD) & -0.23 & $-4.59,4 \cdot 13$ & 0.92 & 51 & 0.66 & $-3.57,4.89$ & 0.75 & 50 \\
\hline \multicolumn{9}{|c|}{ 6-7 years CANTAB DMS total correct (12-s delay) } \\
\hline Early pregnancy (SD) & 0.04 & $-0.08,0.16$ & 0.47 & 393 & 0.06 & $-0.06,0.19$ & 0.32 & 375 \\
\hline Late pregnancy (SD) & -0.06 & $-0.39,0.27$ & 0.73 & 73 & -0.00 & $-0.38,0.37$ & 0.98 & 72 \\
\hline Late-early pregnancy change (SD) & 0.07 & $-0.44,0.58$ & 0.79 & 47 & 0.24 & $-0.42,0.90$ & 0.47 & 46 \\
\hline \multicolumn{9}{|c|}{ 6-7 years CANTAB IED pre-EDS errors ( $z$ score) } \\
\hline Early pregnancy $(\mathrm{SD})$ & 0.02 & $-0.07,0.12$ & 0.65 & 392 & 0.02 & $-0.08,0.12$ & 0.69 & 374 \\
\hline Late pregnancy (SD) & 0.20 & $-0.08,0.47$ & $0 \cdot 16$ & 73 & 0.14 & $-0.18,0.46$ & 0.37 & 72 \\
\hline Late-early pregnancy change (SD) & 0.23 & $-0.19,0.65$ & 0.27 & 47 & 0.01 & $-0.55,0.57$ & 0.96 & 46 \\
\hline \multicolumn{9}{|l|}{$6-7$ years CANTAB IED EDS errors } \\
\hline Early pregnancy $(\mathrm{SD})$ & 0.08 & $-0.02,0.17$ & $0 \cdot 12$ & 392 & 0.06 & $-0.04,0.16$ & 0.22 & 374 \\
\hline Late pregnancy (SD) & 0.11 & $-0.14,0.36$ & 0.38 & 73 & 0.14 & $-0.14,0.43$ & 0.32 & 72 \\
\hline Late-early pregnancy change (SD) & 0.11 & $-0.31,0.52$ & 0.61 & 47 & 0.21 & $-0.34,0.76$ & 0.44 & 46 \\
\hline \multicolumn{9}{|c|}{$6-7$ years CANTAB IED total errors (stage 1 ) in 5 groups } \\
\hline Early pregnancy $(\mathrm{SD})$ & -0.04 & $-0.15,0.07$ & 0.43 & 390 & -0.03 & $-0.15,0.08$ & 0.59 & 372 \\
\hline Late pregnancy (SD) & 0.20 & $-0.13,0.53$ & 0.24 & 73 & 0.23 & $-0.15,0.61$ & 0.23 & 72 \\
\hline Late-early pregnancy change (SD) & 0.57 & $0.08,1.06$ & 0.02 & 47 & 0.64 & $-0.01,1.29$ & 0.05 & 46 \\
\hline \multicolumn{9}{|c|}{$6-7$ years CANTAB IED total errors (stage 8 ) in 5 groups } \\
\hline Early pregnancy (SD) & 0.10 & $-0.03,0.24$ & 0.13 & 390 & 0.07 & $-0.07,0.21$ & 0.33 & 372 \\
\hline Late pregnancy (SD) & 0.17 & $-0.22,0.56$ & 0.40 & 73 & 0.25 & $-0.19,0.69$ & 0.25 & 72 \\
\hline Late-early pregnancy change (SD) & 0.19 & $-0.44,0.82$ & 0.55 & 47 & 0.37 & $-0.49,1.23$ & 0.39 & 46 \\
\hline \multicolumn{9}{|c|}{ 6-7 years CANTAB IED total errors (adjusted) } \\
\hline Early pregnancy (SD) & 0.06 & $-0.03,0.16$ & 0.17 & 392 & 0.04 & $-0.06,0.13$ & 0.46 & 374 \\
\hline Late pregnancy $(\mathrm{SD})$ & 0.27 & $0.00,0.53$ & 0.05 & 73 & 0.27 & $-0.04,0.57$ & 0.08 & 72 \\
\hline Late-early pregnancy change (SD) & 0.24 & $-0.15,0.63$ & 0.22 & 47 & 0.23 & $-0.28,0.75$ & 0.37 & 46 \\
\hline \multicolumn{9}{|c|}{$6-7$ years CANTAB IED stages completed in 4 groups } \\
\hline Early pregnancy (SD) & -0.09 & $-0.18,-0.00$ & 0.04 & 392 & -0.06 & $-0.15,0.03$ & $0 \cdot 16$ & 374 \\
\hline Late pregnancy (SD) & -0.22 & $-0.48,0.03$ & 0.08 & 73 & -0.25 & $-0.53,0.04$ & 0.09 & 72 \\
\hline Late-early pregnancy change (SD) & -0.19 & $-0.58,0.21$ & 0.35 & 47 & -0.32 & $-0.83,0.20$ & 0.22 & 46 \\
\hline \multicolumn{9}{|l|}{ 6-7 years CANTAB SSP span length } \\
\hline Early pregnancy (SD) & -0.04 & $-0.13,0.05$ & 0.35 & 374 & -0.01 & $-0.10,0.08$ & 0.83 & 356 \\
\hline Late pregnancy (SD) & -0.11 & $-0.37,0.15$ & 0.41 & 70 & -0.02 & $-0.29,0.25$ & 0.89 & 69 \\
\hline Late-early pregnancy change (SD) & -0.46 & $-0.88,-0.04$ & 0.03 & 45 & -0.25 & $-0.74,0.24$ & 0.31 & 44 \\
\hline \multicolumn{9}{|c|}{ 6-7 years CANTAB IST mean prob. correct (win condition fixed) in 5 groups } \\
\hline Early pregnancy $(\mathrm{SD})$ & -0.05 & $-0.19,0.10$ & 0.52 & 357 & -0.03 & $-0.18,0.12$ & 0.69 & 340 \\
\hline Late pregnancy (SD) & -0.13 & $-0.80,0.53$ & 0.69 & 27 & 0.33 & $-0.47,1.14$ & 0.40 & 27 \\
\hline Late-early pregnancy change (SD) & -0.19 & $-0.93,0.56$ & 0.60 & 20 & 0.48 & $-0.61,1.56$ & 0.36 & 20 \\
\hline
\end{tabular}

WPPSI, Wechsler Preschool and Primary Scale of Intelligence; IQ, intelligence quotient; WASI, Wechsler Abbreviated Scale of Intelligence; CANTAB, Cambridge Neuropsychological Test Automated Battery; DMS, delayed matching to sample; IED, intra/extra-dimensional shift; EDS, extra-dimensional shift; SSP, Spatial Span; IST, Information Sampling Task.

* Sample sizes varied for specific variables because of outcome-specific missing values. Data were adjusted for maternal BMI, maternal IQ, maternal education, maternal smoking, child's sex and (for CANTAB outcomes) child's age.

accumulation of these fatty acids in the central nervous system. For example, diet quality has been shown to be associated positively with neurodevelopment at the age of 4 years in the present cohort ${ }^{(23)}$, and this may compensate for variations in DHA and ARA status in pregnancy

One possible explanation for the absence of significant associations between maternal ARA and DHA status and neurocognitive outcomes in the children is that the range of concentrations of these fatty acids reported here were sufficient to support normal brain development. Alternatively, it is possible that physiological processes may compensate for low PUFA concentrations in the mothers, thus protecting the development of the fetal brain from any negative effects of sub-optimal accumulation of DHA or ARA. For example, women have greater capacity for DHA synthesis ${ }^{(32)}$ and maintain higher ARA and DHA status than men ${ }^{(33)}$, and thus conversion of essential fatty acids to longer-chain PUFA may compensate for low dietary intakes of preformed DHA and ARA. Furthermore, pregnancy has been associated with a specific increase in DHA in plasma $\mathrm{PC}^{(15,16)}$, which has been shown in animal models to involve changes in the specificity of phospholipid biosynthesis ${ }^{(18)}$ and increased expression of genes involved in conversion of essential fatty acids to longer-chain PUFA ${ }^{(35,36)}$. There is also evidence of biomagnification of DHA by the placenta, leading to a higher concentration in the fetus compared with the mother ${ }^{(37)}$. 
Table 3. Maternal plasma phosphatidylcholine DHA concentration as predictor of cognitive outcomes* (Linear regression coefficients $(\beta)$ and $95 \%$ confidence intervals)

\begin{tabular}{|c|c|c|c|c|c|c|c|c|}
\hline & \multicolumn{4}{|c|}{ Unadjusted } & \multicolumn{4}{|c|}{ Adjusted } \\
\hline & $\beta$ & $95 \% \mathrm{Cl}$ & $P$ & $n$ & $\beta$ & $95 \% \mathrm{Cl}$ & $P$ & $n$ \\
\hline \multicolumn{9}{|l|}{4 years WPPSI IQ } \\
\hline Early pregnancy (SD) & 0.25 & $-2 \cdot 01,2 \cdot 51$ & 0.83 & 146 & -0.03 & $-2 \cdot 29,2 \cdot 23$ & 0.98 & 146 \\
\hline Late pregnancy (SD) & 1.97 & $0.35,3.60$ & 0.02 & 253 & $1 \cdot 13$ & $-0.43,2.69$ & 0.15 & 253 \\
\hline Late-early pregnancy change (SD) & $2 \cdot 10$ & $-0.58,4.78$ & 0.12 & 139 & 1.66 & $-1.04,4.37$ & 0.23 & 139 \\
\hline \multicolumn{9}{|l|}{$6-7$ years WASI } \\
\hline Early pregnancy (SD) & 1.79 & $0.34,3.23$ & 0.02 & 432 & 0.87 & $-0 \cdot 46,2 \cdot 20$ & 0.20 & 414 \\
\hline Late pregnancy $(\mathrm{SD})$ & 1.09 & $-3 \cdot 19,5 \cdot 38$ & 0.61 & 77 & -0.86 & $-5 \cdot 03,3 \cdot 31$ & 0.68 & 76 \\
\hline Late-early pregnancy change (SD) & 1.76 & $-2 \cdot 58,6 \cdot 10$ & 0.42 & 51 & 1.02 & $-3 \cdot 10,5 \cdot 14$ & 0.62 & 50 \\
\hline \multicolumn{9}{|c|}{ 6-7 years CANTAB DMS total correct (12-s delay) } \\
\hline Early pregnancy (SD) & 0.06 & $-0.06,0.18$ & 0.32 & 393 & 0.07 & $-0.06,0.20$ & 0.28 & 375 \\
\hline Late pregnancy $(\mathrm{SD})$ & -0.18 & $-0.51,0.14$ & 0.26 & 73 & -0.15 & $-0.50,0.19$ & 0.38 & 72 \\
\hline Late-early pregnancy change (SD) & -0.04 & $-0.57,0.50$ & 0.89 & 47 & -0.09 & $-0.70,0.52$ & 0.77 & 46 \\
\hline \multicolumn{9}{|c|}{ 6-7 years CANTAB IED pre-EDS errors ( $z$ score) } \\
\hline Early pregnancy (SD) & 0.05 & $-0.05,0.14$ & 0.31 & 392 & 0.07 & $-0.03,0.17$ & 0.18 & 374 \\
\hline Late pregnancy $(\mathrm{SD})$ & 0.23 & $-0.04,0.50$ & 0.09 & 73 & 0.24 & $-0.05,0.53$ & 0.11 & 72 \\
\hline Late-early pregnancy change (SD) & 0.12 & $-0.33,0.56$ & 0.60 & 47 & 0.01 & $-0.50,0.52$ & 0.97 & 46 \\
\hline \multicolumn{9}{|l|}{ 6-7 years CANTAB IED EDS errors } \\
\hline Early pregnancy (SD) & 0.01 & $-0.09,0.10$ & 0.90 & 392 & 0.01 & $-0.09,0.11$ & 0.87 & 374 \\
\hline Late pregnancy $(\mathrm{SD})$ & -0.08 & $-0.33,0.16$ & 0.50 & 73 & -0.07 & $-0.33,0.20$ & 0.61 & 72 \\
\hline Late-early pregnancy change (SD) & -0.12 & $-0.55,0.31$ & 0.57 & 47 & -0.14 & $-0.65,0.37$ & 0.59 & 46 \\
\hline \multicolumn{9}{|c|}{ 6-7 years CANTAB IED total errors (stage 1 ) in 5 groups } \\
\hline Early pregnancy (SD) & -0.06 & $-0.17,0.05$ & 0.28 & 390 & -0.06 & $-0.17,0.06$ & 0.33 & 372 \\
\hline Late pregnancy $(\mathrm{SD})$ & 0.21 & $-0.12,0.53$ & 0.21 & 73 & $0 \cdot 19$ & $-0.16,0.54$ & 0.29 & 72 \\
\hline Late-early pregnancy change (SD) & 0.40 & $-0.13,0.93$ & 0.14 & 47 & 0.35 & $-0.26,0.97$ & 0.25 & 46 \\
\hline \multicolumn{9}{|c|}{ 6-7 years CANTAB IED total errors (stage 8 ) in 5 groups } \\
\hline Early pregnancy (SD) & 0.00 & $-0.14,0.14$ & 0.99 & 390 & -0.01 & $-0 \cdot 15,0 \cdot 13$ & 0.90 & 372 \\
\hline Late pregnancy (SD) & -0.23 & $-0.61,0.15$ & 0.23 & 73 & $-0 \cdot 19$ & $-0.59,0.22$ & 0.37 & 72 \\
\hline Late-early pregnancy change (sD) & -0.36 & $-1.02,0.29$ & 0.27 & 47 & -0.41 & $-1 \cdot 19,0.38$ & 0.30 & 46 \\
\hline \multicolumn{9}{|c|}{ 6-7 years CANTAB IED total errors (adjusted) } \\
\hline Early pregnancy (SD) & 0.03 & $-0.06,0.13$ & 0.49 & 392 & 0.04 & $-0.06,0.13$ & 0.43 & 374 \\
\hline Late pregnancy (SD) & 0.02 & $-0.24,0.29$ & 0.85 & 73 & 0.04 & $-0.24,0.33$ & 0.76 & 72 \\
\hline Late-early pregnancy change (SD) & -0.10 & $-0.52,0.31$ & 0.62 & 47 & -0.17 & $-0.64,0.30$ & 0.47 & 46 \\
\hline \multicolumn{9}{|c|}{ 6-7 years CANTAB IED stages completed in 4 groups } \\
\hline Early pregnancy (SD) & -0.03 & $-0.12,0.05$ & 0.44 & 392 & -0.02 & $-0.11,0.07$ & 0.62 & 374 \\
\hline Late pregnancy (SD) & 0.15 & $-0.10,0.40$ & 0.24 & 73 & 0.13 & $-0.14,0.40$ & 0.34 & 72 \\
\hline Late-early pregnancy change (SD) & 0.19 & $-0.22,0.61$ & 0.35 & 47 & 0.16 & $-0.31,0.64$ & 0.49 & 46 \\
\hline \multicolumn{9}{|l|}{ 6-7 years CANTAB SSP span length } \\
\hline Early pregnancy (SD) & 0.05 & $-0.04,0.14$ & 0.29 & 374 & 0.05 & $-0.04,0.14$ & 0.28 & 356 \\
\hline Late pregnancy $(\mathrm{SD})$ & 0.23 & $-0.02,0.49$ & 0.07 & 70 & 0.19 & $-0.06,0.44$ & 0.13 & 69 \\
\hline Late-early pregnancy change (SD) & -0.27 & $-0.73,0.18$ & 0.24 & 45 & -0.29 & $-0.74,0.15$ & 0.19 & 44 \\
\hline \multicolumn{9}{|c|}{ 6-7 years CANTAB IST mean prob. correct (win condition fixed) in 5 groups } \\
\hline Early pregnancy (SD) & 0.00 & $-0.15,0.15$ & 0.99 & 357 & -0.02 & $-0 \cdot 18,0 \cdot 14$ & 0.79 & 340 \\
\hline Late pregnancy (SD) & 0.12 & $-0.44,0.67$ & 0.67 & 27 & 0.18 & $-0.48,0.84$ & 0.57 & 27 \\
\hline Late-early pregnancy change (SD) & -0.21 & $-1 \cdot 31,0.88$ & 0.68 & 20 & 0.06 & $-1 \cdot 29,1 \cdot 42$ & 0.92 & 20 \\
\hline
\end{tabular}

WPPSI, Wechsler Preschool and Primary Scale of Intelligence; IQ, intelligence quotient; WASI, Wechsler Abbreviated Scale of Intelligence; CANTAB, Cambridge Neuropsychological Test Automated Battery; DMS, delayed matching to sample; IED, intra/extra-dimensional shift; SSP, Spatial Span; IST, Information Sampling Task.

* Sample sizes varied for specific variables because of outcome-specific missing values. Data were adjusted for maternal BMI, maternal IQ, maternal education, maternal smoking, child's sex and (for CANTAB outcomes) child's age.

Strengths of the study include assessment of a range of cognitive outcomes and the availability of measurements of maternal PUFA status in both early and late pregnancy. There was detailed information about potential maternal confounding factors known to influence the cognition of children including maternal education and IQ, smoking and BMI. Limitations of the study include that there was no information about the home environment. Consequently, we were not able to take into account factors that can influence IQ, such as parenting style and the cognitive stimulation of the children. The children who were followed up were a sub-sample of the original cohort and some did not participate in all the tests. As the present findings are from data collected in a cohort study and all the participants with data on fatty acid composition and cognitive function were included in our analysis, we were not able to collect further data to increase sample size; our modest sample size could have contributed to the null findings.

Overall, the findings of this study suggest that maternal ARA and DHA status in early or late pregnancy in the range found in this cohort are unlikely to have major influences on neurocognitive function in the children. Consequently, in this group of healthy children of mothers consuming an omnivorous diet, maternal DHA and ARA status during pregnancy appeared to be adequate for development of cognitive function. 


\section{Acknowledgements}

This work was supported by grants from the Medical Research Council (MC_U147585827, MC_ST_U12055), British Heart Foundation (RG/07/009), Food Standards Agency, NIHR Southampton Biomedical Research Centre, University of Southampton and University Hospital Southampton NHS Foundation Trust and NIHR Musculoskeletal Biomedical Research Unit, University of Oxford. K. M. G., H. M. I., N. C. H. and C. C. are supported by the National Institute for Health Research through the NIHR Southampton Biomedical Research Centre and by the European Union's Seventh Framework Programme (FP7/2007-2013), projects EarlyNutrition and ODIN under grant agreement nos 289346 and 613977. K. M. G. is also supported by the NIHR as an NIHR Senior Investigator (NF-SI-0515-10042) and through the European Union's Erasmus + Capacity-Building ENeA ${ }^{\text {SEA }}$ Project.

The author's responsibilities were as follows: G. C. B. had primary responsibility for the final content of the manuscript; G. C. B., K. M. G. and S. R. C. wrote the manuscript and participated in the design of the study; S. R. C. analysed the data; C. M. S., H. L. F., C. R. G., S. M. R., H. M. I., J. B., N. C. H. and the SWS study group conducted the research; P. C. C., G. C. B., K. M. G. and C. C. oversaw the study. All authors contributed to the interpretation and discussion of the results, and read and approved the final version of the manuscript.

K. M. G. and G. C. B. have received reimbursement for speaking at conferences sponsored by companies selling nutritional products, and are part of an academic consortium that has received research funding from Abbott Nutrition, Nestec and Danone. P. C. C. is a consultant to Danone/Nutricia, DSM, Pronova BioPharma, Cargill and Smartfish and has received speaking honoraria from Danone, DSM, Smartfish and Abbott Nutrition. G. C. B. is an advisor to BASF. None of the other authors had disclosures to report. None of the authors has any conflicts of interest to declare.

\section{Supplementary material}

For supplementary material/s referred to in this article, please visit https://doi.org/10.1017/S0007114518000806

\section{References}

1. Kuipers RS, Luxwolda MF, Offringa PJ, et al. (2012) Fetal intrauterine whole body linoleic, arachidonic and docosahexaenoic acid contents and accretion rates. Prostaglandins Leukot Essent Fatty Acids 86, 13-20.

2. Salem N Jr. \& Pawlosky RJ (1992) Docosahexaenoic acid is an essential nutrient in the nervous system. J Nutr Sci Vitaminol (Tokyo) 38, 153-156.

3. Farquharson J, Jamieson EC, Abbasi KA, et al. (1995) Effect of diet on the fatty acid composition of the major phospholipids of infant cerebral cortex. Arch Dis Child 72, 198-203.

4. Uhl O, Fleddermann M, Hellmuth C, et al. (2016) Phospholipid species in newborn and 4 month old infants after consumption of different formulas or breast milk. PLOS ONE 11, e0162040.
5. Neuringer M, Reisbick S \& Janowsky J (1994) The role of $n-3$ fatty acids in visual and cognitive development: current evidence and methods of assessment. J Pediatr 125, S39-S47.

6. Reisbick S, Neuringer M, Hasnain R, et al. (1994) Home cage behavior of rhesus monkeys with long-term deficiency of omega-3 fatty acids. Physiol Behav 55, 231-239.

7. Lauritzen L, Brambilla P, Mazzocchi A, et al. (2016) DHA effects in brain development and function. Nutrients $\mathbf{8}, 6$.

8. Ghys A, Bakker E, Hornstra G, et al. (2002) Red blood cell and plasma phospholipid arachidonic and docosahexaenoic acid levels at birth and cognitive development at 4 years of age. Early Hum Dev 69, 83-90.

9. Krabbendam L, Bakker E, Hornstra G, et al. (2007) Relationship between DHA status at birth and child problem behaviour at 7 years of age. Prostaglandins Leukot Essent Fatty Acids 76, 29-34.

10. Bakker EC, Ghys AJ, Kester AD, et al. (2003) Long-chain polyunsaturated fatty acids at birth and cognitive function at 7 y of age. Eur J Clin Nutr 57, 89-95.

11. Oken E, Osterdal ML, Gillman MW, et al. (2008) Associations of maternal fish intake during pregnancy and breastfeeding duration with attainment of developmental milestones in early childhood: a study from the Danish National Birth Cohort. Am J Clin Nutr 88, 789-796.

12. Oken E, Radesky JS, Wright RO, et al. (2008) Maternal fish intake during pregnancy, blood mercury levels, and child cognition at age 3 years in a US cohort. Am J Epidemiol 167, $1171-1181$

13. Hibbeln JR, Davis JM, Steer C, et al. (2007) Maternal seafood consumption in pregnancy and neurodevelopmental outcomes in childhood (ALSPAC study): an observational cohort study. Lancet 369, 578-585.

14. Gale CR, Robinson SM, Godfrey KM, et al. (2008) Oily fish intake during pregnancy association with lower hyperactivity but not with higher full-scale IQ in offspring. J Child Psychol Psychiatry 49, 1061-1068.

15. Postle AD, Al MD, Burdge GC, et al. (1995) The composition of individual molecular species of plasma phosphatidylcholine in human pregnancy. Early HumDev 43, 47-58.

16. Otto SJ, Houwelingen AC, Antal M, et al. (1997) Maternal and neonatal essential fatty acid status in phospholipids:an international comparative study. Eur J Clin Nutr 51, 232-242.

17. Meyer BJ, Onyiaodike CC, Brown EA, et al. (2016) Maternal plasma DHA levels increase prior to 29 days post-LH surge in women undergoing frozen embryo transfer: a prospective, observational study of human pregnancy. J Clin Endocrinol Met 101, 1745-1753.

18. Burdge GC, Hunt AN \& Postle AD (1994) Mechanisms of hepatic phosphatidylcholine synthesis in adult rat: effects of pregnancy. Biochem J 303, 941-947.

19. Childs CE, Hoile SP, Burdge GC, et al. (2012) Changes in rat $n-3$ and $n-6$ fatty acid composition during pregnancy are associated with progesterone concentrations and hepatic FADS2 expression. Prostaglandins Leukot Essent Fatty Acids 86, 141-147.

20. Inskip HM, Godfrey KM, Robinson SM, et al. (2006) Cohort profile: The Southampton Women's Survey. Int J Epidemiol 35, 42-48.

21. Burdge GC, Wright $\mathrm{P}$, Jones AE, et al. (2000) A method for separation of phosphatidylcholine, triacylglycerol, nonesterified fatty acids and cholesterol esters from plasma by solid-phase extraction. Br J Nutr 84, 781-787.

22. Lillycrop KA, Costello PM, Teh AL, et al. (2015) Association between perinatal methylation of the neuronal differentiation regulator HES1 and later childhood neurocognitive function and behaviour. Int J Epidemiol 44, 1263-1276. 
23. Gale CR, Martyn CN, Marriott LD, et al. (2009) Dietary patterns in infancy and cognitive and neuropsychological function in childhood. J Child Psychol Psychiatry 50, 816-823.

24. Robbins TW, James M, Owen AM, et al. (1998) A study of performance on tests from the CANTAB battery sensitive to frontal lobe dysfunction in a large sample of normal volunteers: implications for theories of executive functioning and cognitive aging. Cambridge Neuropsychological Test Automated Battery. I Int Neuropsychol Soc 4, 474-490.

25. Rose G (2001) Sick individuals and sick populations. Int J Epidemiol 30, 427-432.

26. Armitage P \& Berry G (2002) Statistical Methods in Medical Research, 3rd ed. Oxford: Blackwell Science Ltd.

27. Greenland S, Pearl J \& Robins JM (1999) Causal diagrams for epidemiologic research. Epidemiology 10, 37-48.

28. Ramakrishnan U, Stinger A, DiGirolamo AM, et al. (2015) Prenatal docosahexaenoic acid supplementation and offspring development at 18 months: randomized controlled trial. PLOS ONE 10, e0120065.

29. Makrides M, Gibson RA, McPhee AJ, et al. (2010) Effect of DHA supplementation during pregnancy on maternal depression and neurodevelopment of young children: a randomized controlled trial. JAMA 304, 1675-1683.

30. Ramakrishnan U, Gonzalez-Casanova I, Schnaas L, et al. (2016) Prenatal supplementation with DHA improves attention at 5 y of age: a randomized controlled trial. Am J Clin Nutr 104, 1075-1082.
31. Delgado-Noguera MF, Calvache JA, Bonfill Cosp X, et al. (2015) Supplementation with long chain polyunsaturated fatty acids (LCPUFA) to breastfeeding mothers for improving child growth and development. Cochrane Database Syst Rev, issue 7, CD007901.

32. Burdge GC \& Wootton SA (2002) Conversion of alpha-linolenic acid to eicosapentaenoic, docosapentaenoic and docosahexaenoic acids in young women. Br J Nutr 88, 411-420.

33. Lohner S, Fekete K, Marosvolgyi T, et al. (2013) Gender differences in the long-chain polyunsaturated fatty acid status: systematic review of 51 publications. Ann Nutr Metab 62, 98-112.

34. Burdge GC \& Postle AD (1994) Hepatic phospholipid molecular species in the guinea pig. Adaptations to pregnancy. Lipids 29, 259-264.

35. Burdge GC, Slater-Jefferies JL, Grant RA, et al. (2008) Sex, but not maternal protein or folic acid intake, determines the fatty acid composition of hepatic phospholipids, but not of triacylglycerol, in adult rats. Prostaglandins Leukot Essent Fatty Acids 78, 73-79.

36. Childs CE, Romeu-Nadal M, Burdge GC, et al. (2010) The polyunsaturated fatty acid composition of hepatic and plasma lipids differ by both sex and dietary fat intake in rats. $J$ Nutr 140, 245-250.

37. Crawford MA, Golfetto I, Ghebremeskel K, et al. (2003) The potential role for arachidonic and docosahexaenoic acids in protection against some central nervous system injuries in preterm infants. Lipids 38, 303-315. 